\title{
O CONE DE VOLATILIDADE NO MERCADO DE OPÇÕES BRASILEIRO
}

\section{THE VOLATILITY CONE IN THE BRAZILIAN OPTIONS MARKET}

Recebido em 15.05.2013. Aprovado em 14.05.2014 Avaliado pelo sistema double blind review

DOI: http://dx.doi.org/10.12712/rpca.v8i2.287

\section{Rodrigo Jatobá Cerqueira}

rjatoba@ig.com.br

Pontifícia Universidade Católica do Rio de Janeiro (PUC-Rio) - Rio de Janeiro - RJ

\section{Antonio Carlos Figueiredo Pinto}

figueiredo@iag.puc-rio.br

Pontifícia Universidade Católica do Rio de Janeiro (PUC-Rio) - Rio de Janeiro - RJ

\section{Marcelo Cabus Klotzle}

klotzle@iag.puc-rio.br

Pontifícia Universidade Católica do Rio de Janeiro (PUC-Rio) - Rio de Janeiro - RJ

\section{Resumo}

Este trabalho tem como objetivo testar se cone de volatilidade aplicado ao mercado de opções brasileiro pode trazer informações adicionais à decisão de compra e venda de volatilidade. Outra contribuição deste trabalho é mostrar se os resultados obtidos nas operações realizadas com o cone de volatilidade variam de acordo com a relação cotação da ação/preço de exercício da opção. Os resultados indicam que a comparação da volatilidade implícita com o cone de volatilidade pode ser um indicador eficiente para prever a evolução da volatilidade futura, permitindo ganhos em operações delta-neutro de compra e de venda de volatilidade. Além disso, foi constatado que existem diferenças nos ganhos, algumas significativas, entre as opções dentro do dinheiro, no dinheiro e fora do dinheiro.

Palavras-chave: Volatilidade implícita. Opções de ações. Cone de volatilidade. Estrutura a termo de volatilidade.

\begin{abstract}
The present study has the objective of testing if the volatility cone applied to the option market can improve the decision process of buying and selling volatility. Another contribution from this study is to evaluate the difference in the results when the volatility cone is used to structure operations, depending on the moneyness of the option. The results indicate that the comparison of implied volatility with the volatility cone can be an efficient indicator to forecast the tendency of future volatility, allowing profits in delta neutral buying and selling volatility strategies. Moreover, there are some indications of differences in profits, some statistically significant, between in the money, at the money and out of the money options.
\end{abstract}

Keywords: Implied volatility. Stock options. Volatility cone. Volatility term structure. 


\section{Introdução}

Burghardt e Morton (1990) são os primeiros a apresentar uma abordagem da análise do comportamento da volatilidade através das distribuições da volatilidade realizada para diversos períodos até o vencimento. Os autores chamam esse método de cone de volatilidade, devido ao formato gráfico em forma de cone, e se deve ao fato da volatilidade implícita para o curto prazo apresentar uma amplitude maior que a para o longo prazo. Hodges e Tompkins (2002) estudam as propriedades do cone de volatilidade e desenvolvem um modelo para eliminação do viés em uma série de dados sobrepostos.

Apesar das diversas possibilidades de utilização do cone de volatilidade a literatura sobre este tema é muito escassa. Na literatura nacional, os estudos realizados sobre a variação da volatilidade analisam somente os efeitos do sorriso de volatilidade, deixando de lado os efeitos da variação de volatilidade diante do tempo de exercício (Junior, 2007; Desterro, 2003; Rodrigues, 2006).

Este trabalho tem como objetivo testar se cone de volatilidade aplicado ao mercado de opções brasileiro pode trazer informações adicionais à decisão de compra e venda de volatilidade. Outra contribuição deste trabalho é mostrar se os efeitos do sorriso de volatilidade persistem diante de operações realizadas com o cone de volatilidade.

Para o calculo do cone de volatilidade foram selecionadas ações da Vale (VALE5) e da Petrobras (PETR4), por serem as únicas ações objeto que apresentam liquidez para séries de diversos prazos de vencimento, diferentes valores de exercício e que são negociadas diariamente. A série obtida é composta dos valores diários de fechamento, abertura, máximo e mínimo dos dois ativos desde janeiro 2005 até junho de 2009. 0 período selecionado contempla dois momentos bastante distintos no mercado financeiro. 0 intervalo de janeiro de 2005 até junho de 2008 reflete um período de estabilidade e grande expectativa de crescimento econômico, já o período seguinte de julho de 2008 até junho de 2009 se caracteriza por de forte crise do sistema bancário mundial.

0 restante do trabalho está organizado da seguinte forma: a seção 2 contém a revisão bibliográfica sobre a modelagem da volatilidade de ativos financeiros ao longo de tempo; a seção 3 apresenta a base de dados utilizada no estudo e os ajustes realizados sobre a mesma, a metodologia empregada na construção do cone de volatilidade e a modelagem usada para avaliar a capacidade de previsão da volatilidade do cone diante de uma série histórica de opções; na seção 4 são apresentados os resultados obtidos; a seção 5 conclui o trabalho, oferecendo considerações finais e possíveis sugestões para trabalhos futuros.

\section{Referencial teórico}

Burghardt e Morton (1990) analisam a variação da distribuição da volatilidade histórica em relação ao tempo até o vencimento. Os modelos de cálculo da volatilidade mais tradicionais baseados na volatilidade histórica utilizam horizontes de tempo fixos para determinação do valor da mesma e com isso não consideram se o tempo até o vencimento da opção em que se quer calcular a volatilidade é de um, dois ou três meses. Diante disso, a comparação da volatilidade implícita com a volatilidade histórica parece inapropriada, uma vez que os horizontes de tempo utilizados no cálculo de cada uma são diferentes.

A comparação da volatilidade com a distribuição da volatilidade histórica para um horizonte de tempo igual ao da volatilidade implícita pode ser um indicador eficiente do comportamento 
futuro da volatilidade. Vender uma volatilidade implícita para um mês a 15\% pode parecer uma boa estratégia se tivermos uma previsão de $12 \%$. Mas pode não parecer uma estratégia tão boa se a volatilidade realizada de um mês tiver apresentado um intervalo de $11 \%$ a $35 \%$. Não é necessária apenas a previsão, mas o entendimento da previsão dentro do contexto de intervalo de volatilidade. Uma maneira simples de fazer isso é através da distribuição da volatilidade em relação ao tempo até o vencimento, também chamada de cone de volatilidade.

Para cada horizonte de tempo até o vencimento são calculadas as variações dos retornos do ativo-objeto e, a partir desta base, são calculados os desvios padrões para um faixa de dados do tamanho do tempo até o vencimento definido. Através de incrementos de defasagem diários, semanais, mensais ou anuais são construídos os valores que compõem a distribuição da volatilidade histórica para o horizonte de tempo definido. Esse mesmo método é aplicado para diversos valores de horizonte de tempo até o vencimento e com isso se obtém uma distribuição da volatilidade histórica para cada faixa de tempo.

A representação gráfica das estatísticas com o valor da volatilidade no eixo das ordenadas e o tempo até o vencimento nas abscissas mostra que a volatilidade para opções de curto prazo apresenta uma amplitude maior do que para opções de longo prazo (Figlewski, 1997). Em virtude desta característica, denomina-se essa figura de cone de volatilidade, devido ao seu formato cônico, com maior amplitude para horizonte de tempo pequeno e seu estreitamento à medida que tempo até o vencimento aumenta.

Em seu artigo, Burghardt e Morton (1990) utilizam dados sobrepostos de 1986 a 1988 para o Iene Japonês para formar os cones de volatilidade. Os autores verificam empiricamente que a volatilidade histórica no curto prazo tem uma maior amplitude que a volatilidade no longo prazo e avaliam a eficiência do modelo através de duas hipóteses: (i) a volatilidade implícita deve ser um previsor sem viés da subseqüente volatilidade realizada, ou seja, se o mercado é eficiente e todas as informações relevantes estão contidas na volatilidade implícita, a distribuição condicional da volatilidade realizada subseqüente é centralizada na volatilidade implícita e (ii) a posição da volatilidade implícita em relação ao cone de volatilidade histórica não deve trazer nenhuma informação sobre a distribuição da volatilidade realizada.

A metodologia utilizada para testar a eficiência do mercado seguiu as seguintes etapas:

- Identificar todas as vezes que a volatilidade implícita era negociada acima do último decil ou abaixo do primeiro decil do cone de volatilidade no período referente ao tempo de vencimento.

- Calcular para opções com 3 a 6 meses do vencimento a volatilidade realizada a partir da data identificada acima até o vencimento.

- Verificar a diferença entre a volatilidade resultante e a volatilidade implícita da opção.

Os resultados obtidos indicam que, para os dias em que a volatilidade implícita estava acima do decil no cone de volatilidade, a previsão da volatilidade realizada foi sistematicamente maior, com uma diferença média de 2,93\%. Ao mesmo tempo, quando a volatilidade implícita estava abaixo do primeiro decil no cone de volatilidade, a volatilidade realizada mostrou-se viesada para baixo, pois a diferença média apresentada foi de 1,71\%. Portanto os resultados mostram que o mercado não é inteiramente eficiente quando a volatilidade implícita é operada em percentuais muito altos ou baixos para opções de longo prazo e o cone de volatilidade pode prover informações se a opção está com preço alto ou baixo. 
Em extensão ao trabalho de Burghardt e Morton (1990), Hodges e Tompkins (2002) descrevem como o cone de volatilidade provê informação sobre as propriedades amostrais da volatilidade para diferentes horizontes. 0 estudo apresenta um modelo para correção do viés gerado pelo uso de dados sobrepostos através da generalização do processo estocástico do ativo objeto.

Os ajustes de viés foram testados através de simulação na qual os preços do ativo objeto foram estimados utilizando quatro modelos alternativos: (i) o movimento geométrico browniano, (ii) distribuição condicional Student-t, (iii) processo de saltos e (iv) distribuição condicional Gaussiana. Para os quatro modelos, foram gerados cem anos de dados em que foram estimadas as propriedades amostrais do cone de volatilidade. As amostras foram então segmentadas em vinte amostras de cinco anos cada e os valores de volatilidade foram determinados para dados sobrepostos. 0 desvio padrão médio da volatilidade das 20 amostras foi comparado com o desvio padrão verdadeiro determinado com a amostra inteira dos 100 anos. Finalmente, o fator de ajuste derivado previamente foi multiplicado pelo desvio padrão médio da volatilidade para avaliar se o viés foi corrigido. 0 estudo propõe então que a variância calculada a partir de uma série de dados sobrepostos precisa ser multiplicada pelo seguinte fator de ajuste:

$$
m=\frac{1}{1-\frac{h}{n}+\frac{h^{2}-1}{3 n^{2}}}
$$

Onde $h$ é o tamanho de cada intervalo de tempo, por exemplo, 20 dias e $n=(T-h)+1$ é o número de intervalos de tempo distintos disponíveis para um total de T observações.

Os autores ainda ressaltam que na comparação entre os modelos e a série empírica do S\&P 500 de 1985 a 2000 foi utilizado o coeficiente de variação, por ser um método geral de comparação e que o modelo que utilizou o processo de volatilidade estocástica capturou melhor a dinâmica observada pela séria empírica do que os processos independentes e identicamente distribuídos, como o movimento geométrico browniano e o processo de salto.

\section{Metodologia Base de Dados}

A base de dados utilizada no estudo é composta de duas categorias: a primeira categoria representa o histórico do preço das ações, utilizado na determinação da volatilidade histórica do ativo objeto para os diferentes horizontes de tempo e conseqüente construção do cone de volatilidade, sendo também um dos parâmetros necessários para o calculo da volatilidade implícita; a segunda categoria refere-se ao histórico do preço das opções e ao histórico da taxa de juros livre de risco, parâmetros fundamentais para a determinação da volatilidade implícita através do cálculo inverso pelo modelo de Black e Scholes (1973).

O levantamento da base de dados dos preços dos ativos foi realizado através do sistema Economática. Este sistema foi escolhido pois, além de armazenar informações de diversas ações em períodos de tempo bastante longos, também possibilita a extração do histórico de preços das ações já ajustados aos pagamentos de proventos. Dessa forma, o cálculo das variações nos preços dos ativos pode ser feito de forma direta sem a necessidade de ajustes adicionais. 
Para o cálculo do cone de volatilidade, foram selecionadas as ações da Vale (VALE5) e da Petrobras (PETR4), por serem as ações objeto únicas a apresentarem liquidez para séries com diversos prazos de vencimento, diferentes valores de exercício e negociações diárias. A série obtida é composta dos valores diários de fechamento, abertura, máximo e mínimo dos dois ativos desde janeiro 2005 até junho de 2009. 0 período selecionado contempla dois momentos bastante distintos no mercado financeiro. 0 intervalo de janeiro de 2005 até junho de 2008 reflete um período de estabilidade e grande expectativa de crescimento econômico, já o período seguinte de julho de 2008 até junho de 2009 se caracteriza por de forte crise do sistema bancário mundial.

A categoria de dados de preços das opções foi obtida através do sítio da Bolsa de Valores, Mercadorias e Futuros e contempla o mesmo período da série acima. As taxas de juros foram obtidas a partir da série DI-PRE no mesmo sítio, esta utilizada como um proxy da taxa de juros livre de risco no calculo das opções. A base utilizada para o calculo da volatilidade implícita é composta das seguintes informações:

- Data de pregão

- Código do ativo

- Preço de fechamento da opção

- Preço de fechamento da ação-objeto

- Preço de exercício

- Data de vencimento

- Dias úteis até o vencimento

- Taxa de juros livre de risco

Com o objetivo de minimizar o viés associados à ausência de sincronia entre os fechamentos no mercado de opções e no mercado de ações, somente opções com pelo menos 100 negociações foram consideradas para as análises. Outro filtro aplicado à base de dados foi o índice de moneyness (cotação da ação/preço de exercício), sendo que somente os contratos perto do dinheiro foram selecionados. Por contratos perto de dinheiro entendem-se aqueles em que a razão entre o valor do ativo-objeto e o preço de exercício está entre 0,95 e 1,05, independente do prazo até o vencimento.

O cone de volatilidade caracteriza a estrutura da volatilidade histórica no tempo através das estatísticas descritivas da distribuição da volatilidade diante dos horizontes de tempo até o vencimento da opção. Diante disso, como apresentado por Burghardt e Morton (1990), a comparação desta estrutura de volatilidade no tempo com a volatilidade implícita pode trazer informações sobre os preços das opções que auxiliem na tomada de decisão de compra e venda.

Uma das principais premissas por trás desta ferramenta é a de que a volatilidade varia ao longo do tempo e que, além disso, segue um processo estocástico de reversão à média. Dessa forma, pode ser feita uma previsão do movimento da volatilidade considerando a posição da mesma em relação ao seu histórico para um mesmo período de tempo até o vencimento.

Diante disso, a metodologia utilizada neste estudo procura abordar o tema através de três etapas. Na primeira etapa foi realizado um teste para validação da premissa da volatilidade implícita seguir um processo estocástico de reversão à média. Para realização deste teste, foi 
utilizado a mesma técnica da raiz unitária apresentada por Dixit et al. (2007). A segunda etapa apresenta o tratamento de dados, as fórmulas e estatísticas utilizadas na construção da estrutura do cone de volatilidade, seguindo a mesma metodologia empregada por Burghardt e Morton (1990). Na terceira etapa, foram realizados os testes de avaliação de eficiência e robustez da ferramenta.

\section{Processo de reversão à media}

Para a realização do teste de reversão à média, foram utilizadas duas séries de dados, uma com a volatilidade implícita para opções com prazo de vencimento até 30 dias e outra com opções de prazo de vencimento entre 30 e 60 dias. O objetivo desta segmentação foi avaliar o comportamento da volatilidade implícita para diferentes prazos de vencimento, uma vez que diversos estudos indicam a diferença de comportamento da volatilidade ao longo do tempo (Stein, 1989; Xu e Taylor, 1994; Dixit, et al., 2007).

O cálculo da volatilidade implícita foi realizado utilizando o método inverso do modelo de Black e Scholes. 0 objetivo foi estimar a volatilidade que, no modelo de Black e Scholes, apresentasse um preço justo para a opção igual ao valor do mercado. Para isso foi desenvolvida uma macro no software Excel com um algoritmo que busca igualar o preço calculado pelo modelo com o do mercado, variando o valor da volatilidade.

No teste da raiz unitária, procurou-se verificar se a série de volatilidade contém ou não uma raiz unitária, ou seja, se é não-estacionária ou estacionária, respectivamente. 0 teste estatístico selecionado para avaliar a hipótese nula foi o teste Augmented Dickey-Fuller (ADF) e sua aplicação realizada com o auxílio do software Eviews.

\section{Cone de Volatilidade}

Na etapa de construção do cone de volatilidade, foi utilizada somente a série de dados dos preços das ações de janeiro de 2005 até janeiro de 2007. As faixas de tempo selecionadas foram de $15,30,45,60,75,90,105$ e 120 dias úteis.

Para a estimativa da volatilidade histórica foram utilizadas as seguintes fórmulas:

$$
\begin{gathered}
\sigma=\sqrt{\frac{1}{N} \sum_{i}\left(X_{i}-\bar{X}\right)^{2}} * \sqrt{252} \\
X_{i+1}=\ln \left(S_{i+1} / S_{i}\right)
\end{gathered}
$$

Onde S é o preço de fechamento da ação e $\mathrm{N}$ é o número de dias de negociação para a faixa de tempo selecionada.

O incremento utilizado para defasar o intervalo de dados no cálculo da volatilidade foi de 15 dias úteis. Com isso, foram obtidos 33 estimadores de volatilidade para a faixa de 15 dias úteis com redução de 1 estimador para cada faixa até a faixa de 120 dias úteis, na qual se obtiveram 26 estimadores. Os estimadores de cada faixa representam os dados para compor sua distribuição. Através da representação gráfica das estatísticas das distribuições se chega ao cone de volatilidade.

No entanto, a série de dados utilizada na construção do cone de volatilidade é composta de dados sobrepostos, ou seja, dados não independentes entre si. Dessa forma os parâmetros de volatilidade calculados a partir da mesma são estimadores enviesados. Neste caso, a principal razão para utilização de dados sobrepostos se deve à necessidade da utilização da quantidade máxima de informação, uma 
que o horizonte de dados já se apresenta reduzido pela segmentação da base para construção do cone e teste de hipótese de eficiência da ferramenta.

Com isso, para corrigir o problema da utilização de dados sobrepostos e eliminar o viés dos estimadores de volatilidade, utilizou-se o fator de correção de Hodges e Tompkins (2002), apresentado anteriormente na equação (1).

\section{Teste de eficiência}

Em um mercado eficiente, a volatilidade implícita contém todas as informações relevantes sobre o curso futuro da volatilidade e deve ser um estimador sem viés da volatilidade realizada subseqüente. Dessa forma, informações sobre o posicionamento da volatilidade implícita em relação à distribuição da volatilidade histórica do cone não deve gerar nenhuma informação sobre a distribuição da volatilidade realizada subseqüente.

Para testar a hipótese de eficiência de mercado, foram utilizados todos os dados das ações da Vale e Petrobrás referentes ao período de janeiro de 2007 até junho de 2009. No entanto, por se tratar de um período marcado por muita instabilidade no cenário econômico mundial, a utilização indiscriminada da série poderia gerar resultados distorcidos. A fim de evitar a distorção nas análises, a base de dados foi segmentada em dois períodos, o primeiro composto de dados de janeiro de 2007 até junho de 2008 e o segundo de julho de 2008 até junho de 2009. Dessa forma, pode-se fazer um teste da ferramenta em dois cenários com características muito distintas: um período de aceleração da economia caracterizado por evolução do mercado de ações sem muita instabilidade e outro período de intensa instabilidade vivido durante a crise mundial.

Assim como em Burghardt e Morton (1990), a metodologia empregada procurou primeiro identificar os dias em que a volatilidade implícita foi negociada com valores acima ou abaixo da média mais duas vezes o desvio padrão. Depois, para opções com prazo de vencimento de até 30 dias e opções com o prazo de vencimento entre 30 e 60 dias, foi calculada a volatilidade realizada nos dias restantes até o vencimento de cada opção para então subtraí-la da volatilidade implícita da opção.

Este procedimento simula o resultado obtido com a realização de uma operação de compra de volatilidade, compra de uma opção e posição de delta-hedge até o vencimento, nos casos em que volatilidade implícita esteja abaixo dos parâmetros de referência acima indicados, ou venda de volatilidade, com a venda de uma opção e posição de delta-hedge nos casos em que a volatilidade se apresenta acima do parâmetro de referência.

A análise da série de dados, além do objetivo principal deste trabalho que é a avaliação da capacidade do cone de volatilidade em trazer informações adicionais que auxiliem os participantes do mercado na decisão de compra e venda de volatilidade, também procura avaliar questões secundárias, mas de grande importância. Estas incluem a análise do desempenho do cone para opções com diferentes preços de exercício, classificadas como opções dentro do dinheiro, no dinheiro e fora do dinheiro e opções de curto e médio prazo.

Na análise do cone de volatilidade para opções com diferentes preços de exercício, aplicou-se sobre a base de dados filtrada pelo índice de moneyness (cotação da ação/preço de exercício) entre 0,95 e 1,05, a seguinte classificação: 
- Opções fora do dinheiro: opções com índice de moneyness menor que 0,975

- Opções no dinheiro: opções com índice de moneyness entre 0,975 e 1,025

- Opções dentro do dinheiro: opções com índice de moneyness maiores que 1,025.

A partir desta classificação, procurou-se avaliar a diferença média entre os tipos de opção para os eventos em que a volatilidade implícita esteve respectivamente acima ou abaixo do limite superior e inferior do cone de volatilidade. Para avaliar a igualdade estatística entre as médias utilizou-se o teste F de ANOVA.

Com isso, foram realizadas simulações para cada uma dessas novas bases e coletados os resultados para eventos de volatilidade respectivamente acima e abaixo do topo e do vale do cone de volatilidade. A comparação entre o desempenho desses dois tipos de opção foi também foi feito através do teste $\mathrm{F}$ de ANOVA.

As análises propostas acima buscam portanto apresentar o cone de volatilidade e suas características para opções da Vale e Petrobras, avaliar o desempenho de utilização do cone sobre a série histórica das opções e entender se existe alguma distinção devido a características relacionadas ao preço de exercício e tempo até o vencimento das opções. Além disso, procurouse também avaliar o comportamento do cone de volatilidade diante de uma série histórica marcada por elementos de extrema incerteza do mercado, como a crise de 2008.

\section{Resultados \\ Teste de raiz unitária}

Uma das principais premissas do cone de volatilidade é de que a volatilidade implícita segue um processo estocástico de reversão à média. Dessa forma, toda vez que a volatilidade implícita ultrapassar o limite superior ou inferior da distribuição da volatilidade histórica para cada horizonte de tempo até o vencimento, há uma tendência de a mesma retornar à média de longo prazo.

As tabelas abaixo apresentam o teste da raiz unitária para avaliar se as séries temporais da volatilidade implícita seguem um processo estocástico de reversão à média. A estatística de teste utilizada foi a ADF (Augmented Dickey-Fuller) com nível de significância de 5\%.

\begin{tabular}{|l|c|}
\hline ADF (estatítica de teste) & $-3,190$ \\
\hline Prob. (p-valor) & 0,021 \\
\hline Valor Crítico (5\%) & $-2,869$ \\
\hline
\end{tabular}

Tabela 1 - Teste da raiz unitária para a volatilidade implícita de opções da Vale Fonte: elaboração própria.

\begin{tabular}{|l|c|}
\hline ADF (estatística de teste) & $-4,255$ \\
\hline Prob (p-valor) & 0,001 \\
\hline Valor crítico (5\%) & $-2,866$ \\
\hline
\end{tabular}

Tabela 2 - Teste da raiz unitária para a volatilidade implícita de opções da Petrobras Fonte: elaboração própria.

De acordo com os testes acima, se rejeita a hipótese nula da raiz unitária. Com isso, pode-se 
concluir que a volatilidade implícita segue um processo estocástico de reversão à média, validando dessa forma a premissa do modelo.

\section{Cone de volatilidade para opções da Vale}

A tabela abaixo apresenta os valores de volatilidade para horizontes de tempo que vão desde 15 dias úteis até 120 dias úteis.

$\begin{array}{lcccccccc}\text { Data } & 15 & 30 & 45 & 60 & 75 & 90 & 105 & 12 \\ 24 / 1 / 2005 & 36,3 \% & & & & & & & \\ 17 / 2 / 2005 & 19,6 \% & 29,6 & & & & & & \\ 10 / 3 / 2005 & 43,6 \% & 34,4 & 34,7 \% & & & & & \\ 1 / 4 / 2005 & 42,1 \% & 43,4 & 37,1 \% & 36,8 \% & & & & \\ 25 / 4 / 2005 & 37,9 \% & 39,4 & 41,8 \% & 37,4 \% & 37,3 \% & & & \\ 16 / 5 / 2005 & 41,6 \% & 39,1 & 39,7 \% & 41,6 \% & 38,1 \% & 38,0 \% & & \\ 7 / 6 / 2005 & 36,6 \% & 39,3 & 38,6 \% & 39,2 \% & 40,6 \% & 37,8 \% & 37,7 \% & \\ 28 / 6 / 2005 & 30,6 \% & 33,2 & 36,3 \% & 36,6 \% & 37,5 \% & 38,9 \% & 36,8 \% & 36,8 \% \\ 19 / 7 / 2005 & 26,0 \% & 28,1 \% & 30,8 \% & 34,0 \% & 34,9 \% & 36,0 \% & 37,4 \% & 35,6 \% \\ 9 / 8 / 2005 & 21,1 \% & 23,3 \% & 26,0 \% & 28,6 \% & 32,0 \% & 33,3 \% & 34,5 \% & 35,9 \% \\ 30 / 8 / 2005 & 25,4 \% & 23,6 \% & 24,1 \% & 25,8 \% & 28,0 \% & 30,9 \% & 32,1 \% & 33,4 \% \\ 21 / 9 / 2005 & 27,9 \% & 27,7 \% & 25,5 \% & 25,4 \% & 26,5 \% & 28,2 \% & 30,8 \% & 32,0 \% \\ 13 / 10 / 2005 & 40,4 \% & 36,0 \% & 32,7 \% & 30,2 \% & 29,3 \% & 29,4 \% & 30,3 \% & 32,2 \% \\ 4 / 11 / 2005 & 37,8 \% & 39,2 \% & 36,2 \% & 33,8 \% & 31,6 \% & 30,6 \% & 30,6 \% & 31,2 \% \\ 28 / 11 / 2005 & 21,1 \% & 30,4 \% & 34,0 \% & 33,1 \% & 31,6 \% & 30,1 \% & 29,5 \% & 29,5 \% \\ 19 / 12 / 2005 & 26,6 \% & 23,6 \% & 29,1 \% & & & & & \end{array}$

Tabela 3 - Histograma de volatilidade histórica da VALE5 para intervalos de tempo de $15,30,45,60,75,90,105$ e 120 dias úteis

Fonte: elaboração própria.

As distribuições de volatilidade para os diferentes horizontes de tempo apresentam características similares de assimetria e curtose, com exceção da faixa de 120 dias úteis que apresentaram uma assimetria à esquerda ou negativa.

Assim como em Burghart e Morton (1990), a volatilidade realizada no curto prazo apresenta maior variabilidade do que a volatilidade no longo prazo. A volatilidade calculada com horizonte de tempo de 15 dias úteis apresentou um valor máximo de 43,6\% e um valor mínimo de 18,1\%, uma amplitude de 25,5\%. Enquanto isso, para um horizonte de tempo até 120 dias úteis até o vencimento, o valor máximo da volatilidade histórica foi de 36,8\% e um valor mínimo de 25,5\%, uma amplitude de $11,3 \%$. Esse comportamento reforça a premissa avaliada na literatura sobre a estrutura a termo da volatilidade de que novas informações no mercado causam maior impacto na volatilidade de opções no curto prazo do que opções de longo prazo.

A evidência da diferença significativa entre as volatilidades no curto prazo e longo prazo revela que avaliações de preço de opções baseadas em métodos de cálculo de volatilidade com base em períodos fixos podem trazer grandes erros por não considerarem as reais amplitudes da volatilidade de acordo com o prazo até o vencimento. Esse resultado é importante não só para avaliação de preço de opções, mas também na gestão de carteira e risco, pois permite que seja feita uma modelagem de risco mais adequada às variações temporais. Nesse sentido, o cone de volatilidade suporta de maneira simples o entendimento dos valores extremos que a opção pode alcançar ao representar as características da volatilidade ao longo do tempo até o vencimento.

Além disto, a maior utilidade do cone de volatilidade está em indicar se o valor da volatilidade implícita para uma série de opções está acima ou abaixo das estatísticas de variabilidade da distribuição da volatilidade para um determinado período de dias até o vencimento. Isto é 
importante pois permite antecipar importantes erros de previsão que poderiam ocorrer por não se contabilizar a verdadeira estrutura temporal da volatilidade.

Entender esses desvios da volatilidade da média histórica se mostra primordial no campo prático, pois é comprovado empiricamente no campo das Finanças Comportamentais que muitos tomadores de decisões financeiras não se comportam de maneira racional, ou seja, estão sujeitos a diversos vieses cognitivos na hora de decidir como investir ou prever o comportamento do preço dos ativos (Ackert e Deaves, 2010). A partir do momento em que se conhecem os desvios dos preços dos ativos do que seria seu apreçamento justo, fica mais fácil orientar os negociadores de opções em seu processo de tomada decisão.

Para dar mais robustez à análise, o mesmo método foi aplicado sobre as séries de opções presentes na base dados no período de 02 de janeiro de 2007 até 30 de junho de 2009. Como citado na metodologia, a série de dados foi segmentada em duas novas séries compreendidas no período de 02 de janeiro de 2007 até 30 de junho de 2008 e 01 de julho de 2008 até 30 de junho de 2009. O objetivo desta segmentação foi avaliar a utilização do cone diante de duas condições de mercado diferenciadas e verificar qual o resultado da ferramenta em um teste de estresse caracterizado por um período de extrema incerteza iniciado no segundo semestre de 2008 com a crise de crédito dos bancos americanos. Foram considerados na análise somente os percentuais de volatilidade referente aos primeiros 44 dias até o vencimento. Essa restrição se deve ao fato das opções só apresentarem liquidez com dois meses até o vencimento.

Além disso, foram retiradas da amostra opções que apresentaram um índice de moneyness menor do que 0,95 e maior do 1,05 no primeiro dia de operação. Com isso, se eliminaram as séries de opções com valores de exercício muito distantes do preço da ação, reduzindo dessa forma a utilização de valores de volatilidade muito discrepantes, o que poderia enviesar os resultados.

A tabela abaixo apresenta os resultados encontrados para os eventos de compra e venda de volatilidade toda vez que a volatilidade implícita alcançou o limite superior determinado pelo percentil $90 \%$ da distribuição de volatilidade para cada horizonte de tempo ou o limite inferior determinado pelo percentil $10 \%$ da mesma distribuição de volatilidade.

\begin{tabular}{lcc}
\hline Resumo Estatístico & $\begin{array}{c}\text { Volatilidade Implicita no topo } \\
\text { do cone de volatilidade* }\end{array}$ & $\begin{array}{c}\text { Volatilidade Implicita no vale } \\
\text { do cone de volatilidade** }\end{array}$ \\
\hline Máximo & $50,0 \%$ & $42 \%$ \\
Percentil 90\% & $32,0 \%$ & $26,0 \%$ \\
Percentil 10\% & $-14,6 \%$ & $-2,0 \%$ \\
Mínimo & $-40,0 \%$ & $-24,0 \%$ \\
Média & $6,6 \%$ & $9,5 \%$ \\
Desvio - padrão & $18 \%$ & $12 \%$ \\
T-value & 2,50 & 5,49 \\
Sig. & 0,016 & 0,000 \\
Número de observações & 49 & 49 \\
\hline
\end{tabular}

*Percentil $90 \%$

${ }^{* *}$ Percentil $10 \%$

Tabela 4 - Variação entre a volatilidade implícita ao atingir níveis maiores ou menores que o cone de volatilidade e a volatilidade realizada a partir da data de comparação até o vencimento das opções da Vale.

Fonte: elaboração própria.

Nos dias em que a volatilidade implícita foi negociada acima do limite superior do cone de 
volatilidade, na média haveria lucro, se uma posição de venda de volatilidade fosse aberta, pois a volatilidade realizada acabou sendo 6,6 pontos percentuais abaixo da volatilidade implícita no início da operação. A análise de hipótese de que a média dos resultados é menor ou igual a zero ao nível de significância de 5\% indica que a hipótese nula pode ser rejeitada, ou seja, a média da variação da volatilidade pode ser considerada maior que zero.

Além disso, quando a volatilidade implícita foi menor que o limite inferior do cone de volatilidade, os resultados também mostram que a utilização do cone de volatilidade poderia, na média, trazer lucro, se uma posição de compra de volatilidade fosse aberta, pois a volatilidade realizada acabou sendo 9,5 pontos percentuais acima da volatilidade implícita no início da operação. Novamente, o ganho médio maior que zero é estatisticamente significativo ao nível de significância de $1 \%$.

Esses resultados mostram que o mercado de opções não é inteiramente eficiente, pelo menos quando a volatilidade implícita é negociada em percentuais muito altos ou muito baixos. Isto vai de encontro com o que foi argumentado anteriormente, de que os agentes econômicos não se comportam de maneira racional na hora de tomar decisões de cunho financeiro. Baseado nisto, pode-se afirmar que o cone de volatilidade consegue dar uma informação bastante útil e confiável sobre se as opções estão caras ou baratas e orientar os tomadores de decisões na hora de investir nestes ativos, seja para fins especulativos ou de gestão do risco.

Após a constatação desses resultados, a próxima tarefa foi identificar se existe diferença de desempenho na utilização do cone de volatilidade entre opções dentro do dinheiro, no dinheiro e fora do dinheiro. Essa análise é importante, uma vez que relaciona os efeitos do fenômeno do sorriso de volatilidade com a variação da volatilidade no tempo. Procura-se entender então se as distorções na volatilidade implícita causadas pelo efeito do sorriso de volatilidade reforçam as indicações de oportunidade de compra e venda para as opções. Esta análise dá portanto mais robustez às constatações feitas anteriormente sobre a aplicabilidade do modelo para a tomada de decisões no âmbito de negociações com opções.

A tabela abaixo apresenta um resumo dos resultados encontrados para cada tipo de opção. Utilizando a mesma metodologia acima, as variações da volatilidade foram calculadas toda vez que a volatilidade implícita ultrapassou o limite superior ou inferior do cone de volatilidade.

\begin{tabular}{llcc}
\hline & $\begin{array}{l}\text { Resumo } \\
\text { Estatístico }\end{array}$ & $\begin{array}{l}\text { Volatilidade Implicita no topo } \\
\text { do cone de volatilidade* }\end{array}$ & $\begin{array}{c}\text { Volatilidade Implicita no vale } \\
\text { do cone de volatilidade** }\end{array}$ \\
\hline fora do dinheiro & máximo & $12 \%$ & $16 \%$ \\
& média & $-6 \%$ & $7 \%$ \\
& mínimo & $-39,8 \%$ & $-2 \%$ \\
& \# observacões & 16 & 2 \\
\hline no dinheiro & máximo & $30 \%$ & $13 \%$ \\
& média & $5 \%$ & $3 \%$ \\
& mínimo & $-17 \%$ & $-11 \%$ \\
& \# observações & 6 & 18 \\
\hline dentro do dinheiro & máximo & $41 \%$ & $42 \%$ \\
& média & $14 \%$ & $15 \%$ \\
& mínimo & $-10 \%$ & $-16 \%$ \\
Estatística F & \# observações & 27 & 29 \\
Sig. & & 8,083 & 16,237 \\
\hline
\end{tabular}

Tabela 5 - Variação entre a volatilidade implícita ao atingir níveis superiores ou inferiores ao cone de volatilidade e a volatilidade realizada até o exercício para opções da Vale fora do dinheiro, no dinheiro e dentro do dinheiro.

Fonte: elaboração própria.

As opções fora do dinheiro tiveram em média uma variação negativa de 6\% para os eventos em 
que a volatilidade implícita ultrapassou o limite superior do cone de volatilidade. Neste caso operações realizadas a partir da indicação do cone de volatilidade teriam em média prejuízo, pois a volatilidade realizada acabou sendo 6,0 pontos percentuais acima da volatilidade implícita no início da operação. Nos eventos em que a volatilidade implícita foi menor que o limite inferior, as operações de compra de volatilidade teriam dado lucro em média, pois a volatilidade realizada acabou sendo 7,0 pontos percentuais acima da volatilidade implícita no início da operação. Entretanto, apesar deste resultado positivo no segundo caso, o mesmo deve ser analisado com cautela, devido ao número muito pequeno de observações contabilizadas.

Ao contrario das opções fora do dinheiro, opções no dinheiro tiveram em média uma variação positiva de $5 \%$ ao atingirem o topo do cone de volatilidade e de $3 \%$ quando a volatilidade implícita esteve menor que o limite inferior do cone. No entanto, o melhor desempenho encontrado foi o das opções dentro do dinheiro. Além de apresentarem um número maior de eventos, com 27 observações que ultrapassaram o topo e 29 observações que ultrapassaram o vale, as opções dentro do dinheiro apresentaram uma média de $14 \%$ e $15 \%$ para variações no topo e no vale respectivamente.

A análise de variância entre a média dos três tipos de opção confirma que, ao nível de significância de 1\%, pode-se rejeitar a hipótese nula de que as médias são iguais. 0 teste de Turkey, realizado através do software SPSS, confirma que além de existir diferença significativa entre as médias, a média das opções dentro do dinheiro é maior do que a média das opções fora do dinheiro ao nível de significância de 1\%. No entanto não se pode garantir estatisticamente que média das opções dentro do dinheiro seja maior que as opções no dinheiro.

A comparação entre as opções com diferentes preços de exercício mostra que o cone de volatilidade não apresenta resultados positivos para as opções fora do dinheiro e que para opções no dinheiro e dentro do dinheiro a utilização do cone de volatilidade pode apresentar resultados positivos. No entanto, para os três casos existem riscos de erro na previsão do movimento da volatilidade que podem levar a perdas, conforme mostrado na Tabela 5. Isto mostra que, quando se investe em opções, é importante classificá-las entre os diferentes preços de exercício.

No período de 01 de julho de 2008 até 30 de junho de 2009, que foi caracterizado pela crise de crédito dos bancos americanos, os resultados obtidos apresentaram grande variabilidade e um rendimento médio baixo ou negativo. Os testes estatísticos não rejeitam a hipótese de que a variação média da volatilidade ao atingir os limites do cone é menor ou igual que zero. A grande volatilidade do mercado no período analisado se evidencia através da pequena quantidade de eventos em que a volatilidade implícita esteve abaixo do limite mínimo do cone de volatilidade, indicando que a mesma apresentou na maioria das vezes percentuais elevados de volatilidade implícita. Os resultados encontrados apontam então para uma limitação de uso do cone de volatilidade diante de um mercado em período de grande incerteza.

\section{Cone de volatilidade para opções da Petrobras}

A tabela 6 apresenta a distribuição da volatilidade histórica da PETR4 para os horizontes de tempo de 15 até 120 dias úteis de janeiro de 2005 até junho de 2007.

As distribuições apresentam assimetria positiva leve em todos os casos, com exceção da distribuição para o prazo de vencimento de 120 dias úteis, que apresentou assimetria levemente negativa.

O perfil cônico é caracterizado pela variação da amplitude de acordo com o tempo até o 
vencimento. A volatilidade histórica para um intervalo de 120 dias úteis apresentou um valor máximo de $34,9 \%$ e mínimo de $26,1 \%$, resultando em uma amplitude de 8,8\%. Esse percentual aumenta com a redução do tempo de intervalo e chega a $39 \%$ quando o prazo até o vencimento é de 15 dias úteis. Nesse caso, o valor máximo da volatilidade foi de 52,1\% e o mínimo de $13 \%$, levando a uma amplitude de $39,1 \%$.

\begin{tabular}{|c|c|c|c|c|c|c|c|c|}
\hline Data & 15 & 30 & 45 & 60 & 75 & 90 & 105 & 120 \\
\hline $24 / 1 / 05$ & $16 \%$ & & & & & & & \\
\hline $17 / 2 / 05$ & $24 \%$ & $21 \%$ & & & & & & \\
\hline $10 / 3 / 05$ & $36 \%$ & $30 \%$ & $27 \%$ & & & & & \\
\hline $1 / 4 / 05$ & $29 \%$ & $33 \%$ & $30 \%$ & $27 \%$ & & & & \\
\hline $25 / 4 / 05$ & $28 \%$ & $28 \%$ & $31 \%$ & $30 \%$ & $28 \%$ & & & \\
\hline $16 / 5 / 05$ & $30 \%$ & $29 \%$ & $29 \%$ & $31 \%$ & $30 \%$ & $28 \%$ & & \\
\hline $7 / 6 / 05$ & $26 \%$ & $28 \%$ & $28 \%$ & $28 \%$ & $30 \%$ & $29 \%$ & $28 \%$ & \\
\hline $28 / 6 / 05$ & $24 \%$ & $24 \%$ & $27 \%$ & $27 \%$ & $28 \%$ & $29 \%$ & $28 \%$ & $27 \%$ \\
\hline $19 / 7 / 05$ & $25 \%$ & $25 \%$ & $25 \%$ & $26 \%$ & $27 \%$ & $27 \%$ & $28 \%$ & $28 \%$ \\
\hline $9 / 8 / 05$ & $22 \%$ & $24 \%$ & $24 \%$ & $24 \%$ & $25 \%$ & $26 \%$ & $27 \%$ & $28 \%$ \\
\hline $30 / 8 / 05$ & $26 \%$ & $24 \%$ & $25 \%$ & $24 \%$ & $24 \%$ & $26 \%$ & $26 \%$ & $27 \%$ \\
\hline $21 / 9 / 05$ & $23 \%$ & $25 \%$ & $24 \%$ & $25 \%$ & $24 \%$ & $24 \%$ & $25 \%$ & $26 \%$ \\
\hline $13 / 10 / 05$ & $37 \%$ & $35 \%$ & $32 \%$ & $30 \%$ & $29 \%$ & $28 \%$ & $28 \%$ & $28 \%$ \\
\hline 4/11/05 & $52 \%$ & $46 \%$ & $41 \%$ & $38 \%$ & $35 \%$ & $34 \%$ & $32 \%$ & $32 \%$ \\
\hline $28 / 11 / 05$ & $34 \%$ & $43 \%$ & $42 \%$ & $39 \%$ & $37 \%$ & $35 \%$ & $34 \%$ & $32 \%$ \\
\hline $19 / 12 / 05$ & $16 \%$ & $27 \%$ & $36 \%$ & $38 \%$ & $36 \%$ & $34 \%$ & $33 \%$ & $32 \%$ \\
\hline 10/1/06 & $24 \%$ & $20 \%$ & $26 \%$ & $34 \%$ & $36 \%$ & $34 \%$ & $33 \%$ & $32 \%$ \\
\hline $1 / 2 / 06$ & $25 \%$ & $24 \%$ & $22 \%$ & $25 \%$ & $32 \%$ & $34 \%$ & $33 \%$ & $32 \%$ \\
\hline $22 / 2 / 06$ & $40 \%$ & $34 \%$ & $31 \%$ & $28 \%$ & $29 \%$ & $34 \%$ & $35 \%$ & $34 \%$ \\
\hline $17 / 3 / 06$ & $35 \%$ & $37 \%$ & $34 \%$ & $32 \%$ & $30 \%$ & $30 \%$ & $34 \%$ & $35 \%$ \\
\hline 7/4/06 & $24 \%$ & $30 \%$ & $33 \%$ & $32 \%$ & $31 \%$ & $29 \%$ & $29 \%$ & $33 \%$ \\
\hline $3 / 5 / 06$ & $25 \%$ & $24 \%$ & $28 \%$ & $31 \%$ & $31 \%$ & $30 \%$ & $28 \%$ & $29 \%$ \\
\hline 24/5/06 & $23 \%$ & $25 \%$ & $25 \%$ & $27 \%$ & $30 \%$ & $30 \%$ & $29 \%$ & $28 \%$ \\
\hline $14 / 6 / 06$ & $44 \%$ & $34 \%$ & $33 \%$ & $31 \%$ & $31 \%$ & $33 \%$ & $32 \%$ & $32 \%$ \\
\hline $6 / 7 / 06$ & $33 \%$ & $40 \%$ & $36 \%$ & $33 \%$ & $32 \%$ & $32 \%$ & $33 \%$ & $33 \%$ \\
\hline $27 / 7 / 06$ & $34 \%$ & $33 \%$ & $38 \%$ & $35 \%$ & $33 \%$ & $32 \%$ & $32 \%$ & $33 \%$ \\
\hline $17 / 8 / 06$ & $19 \%$ & $27 \%$ & $30 \%$ & $34 \%$ & $32 \%$ & $31 \%$ & $30 \%$ & $31 \%$ \\
\hline $8 / 9 / 06$ & $26 \%$ & $23 \%$ & $27 \%$ & $29 \%$ & $33 \%$ & $31 \%$ & $31 \%$ & $30 \%$ \\
\hline 29/9/06 & $36 \%$ & $31 \%$ & $27 \%$ & $29 \%$ & $30 \%$ & $33 \%$ & $32 \%$ & $31 \%$ \\
\hline $23 / 10 / 06$ & $30 \%$ & $33 \%$ & $31 \%$ & $28 \%$ & $29 \%$ & $30 \%$ & $33 \%$ & $32 \%$ \\
\hline $14 / 11 / 06$ & $25 \%$ & $28 \%$ & $31 \%$ & $30 \%$ & $28 \%$ & $29 \%$ & $30 \%$ & $32 \%$ \\
\hline $7 / 12 / 06$ & $21 \%$ & $23 \%$ & $26 \%$ & $28 \%$ & $28 \%$ & $27 \%$ & $28 \%$ & $29 \%$ \\
\hline $2 / 1 / 07$ & $13 \%$ & $17 \%$ & $20 \%$ & $23 \%$ & $26 \%$ & $26 \%$ & $25 \%$ & $26 \%$ \\
\hline 23/1/07 & $33 \%$ & $26 \%$ & $25 \%$ & $25 \%$ & $26 \%$ & $28 \%$ & $28 \%$ & $27 \%$ \\
\hline Máximo & $52,1 \%$ & $45,7 \%$ & $41,9 \%$ & $38,9 \%$ & $36,7 \%$ & $34,8 \%$ & $35,1 \%$ & $34,9 \%$ \\
\hline +1 desvio & $36,4 \%$ & $35,4 \%$ & $34,7 \%$ & $33,9 \%$ & $33,3 \%$ & $33,1 \%$ & $33,0 \%$ & $32,8 \%$ \\
\hline media & $28,2 \%$ & $28,9 \%$ & $29,4 \%$ & $29,8 \%$ & $30,0 \%$ & $30,1 \%$ & $30,2 \%$ & $30,3 \%$ \\
\hline -1 desvio & $20,1 \%$ & $22,4 \%$ & $24,1 \%$ & $25,6 \%$ & $26,7 \%$ & $27,1 \%$ & $27,4 \%$ & $27,7 \%$ \\
\hline Minimo & $13,0 \%$ & $17,2 \%$ & $20,1 \%$ & $23,1 \%$ & $24,3 \%$ & $24,4 \%$ & $25,4 \%$ & $26,1 \%$ \\
\hline Amplitude & $39,0 \%$ & $28,5 \%$ & $21,8 \%$ & $15,9 \%$ & $12,3 \%$ & $10,4 \%$ & $9,7 \%$ & $8,8 \%$ \\
\hline Coeficiente & $28,8 \%$ & $22,5 \%$ & $18,0 \%$ & $13,9 \%$ & $11,0 \%$ & $9,8 \%$ & $9,3 \%$ & $8,5 \%$ \\
\hline Curtose & $-1,148$ & $-0,708$ & $-0,941$ & $-0,867$ & $-0,865$ & $-0,822$ & $-0,685$ & $-0,830$ \\
\hline Assimetria & 0,108 & 0,406 & 0,255 & 0,253 & 0,331 & 0,367 & 0,197 & $-0,024$ \\
\hline Percentil 10\%' & $20 \%$ & $23 \%$ & $24 \%$ & $25 \%$ & $26 \%$ & $26 \%$ & $27 \%$ & $27 \%$ \\
\hline Percentil 90\%" & $37 \%$ & $36 \%$ & $36 \%$ & $35 \%$ & $35 \%$ & $34 \%$ & $33 \%$ & $33 \%$ \\
\hline
\end{tabular}

Tabela 6 - Histograma de volatilidade histórica da PETR4 para intervalos de tempo de $15,30,45,60,75,90,105$ e 120 dias úteis

Fonte: elaboração própria

A tabela 7 apresenta os resultados encontrados para os eventos de compra e venda de volatilidade toda vez que a volatilidade implícita alcançou o limite superior determinado pelo percentil $90 \%$ ou o limite inferior determinado pelo percentil $10 \%$ da distribuição de volatilidade para cada horizonte de tempo. 


\begin{tabular}{|c|c|c|}
\hline Resumo Estatístico & $\begin{array}{l}\text { Volatilidade Implicita no topo do } \\
\text { cone de volatilidade* }\end{array}$ & $\begin{array}{l}\text { Volatilidade Implicita no vale do } \\
\text { cone de volatilidade** }\end{array}$ \\
\hline Máximo & $34,2 \%$ & $92 \%$ \\
\hline Percentil 90\% & $16,3 \%$ & $17,0 \%$ \\
\hline Percentil $10 \%$ & $-6,9 \%$ & $-7,6 \%$ \\
\hline Mínimo & $-14,9 \%$ & $-10,4 \%$ \\
\hline Média & $5,1 \%$ & $8,9 \%$ \\
\hline Desvio - padrão & $11 \%$ & $17 \%$ \\
\hline T-value & 2,53 & 3,37 \\
\hline Sig. & 0,019 & 0,002 \\
\hline Número de observações & 27 & 40 \\
\hline
\end{tabular}

\section{*Percentil $90 \%$}

$* *$ Percentil $10 \%$

Tabela 7 - Variação entre a volatilidade implícita ao atingir níveis maiores ou menores que o cone de volatilidade e a volatilidade realizada a partir da data de comparação até o vencimento das opções da Petrobrás.

Fonte: elaboração própria

No período de janeiro de 2007 até junho de 2008, ocorreram 27 eventos nos quais a volatilidade implícita das séries analisadas esteve acima do limite superior do cone de volatilidade e 40 casos nos quais a mesma esteve abaixo do limite inferior do cone.

Nas operações de venda de volatilidade, ou seja, quando a volatilidade implícita esteve maior que o limite superior do cone, em média a volatilidade realizada acabou sendo 5,1 pontos percentuais abaixo da volatilidade implícita no início da operação. Ao nível de significância de $5 \%$, pode-se rejeitar a hipótese nula de que a variação média nesta operação seja menor ou igual que zero.

Nos casos em que a volatilidade implícita esteve abaixo do limite inferior do cone de volatilidade, em média a volatilidade realizada acabou sendo 8,9 pontos percentuais acima da volatilidade implícita no início da operação. Assim como no resultado anterior, pode-se rejeitar a hipótese nula da média ser menor ou igual a zero ao nível de significância de $5 \%$.

Após a constatação desses resultados, a próxima tarefa foi identificar se existe diferença de desempenho na utilização do cone de volatilidade entre opções dentro do dinheiro, no dinheiro e fora do dinheiro.

A tabela 8 apresenta o desempenho do cone de volatilidade para opções com moneyness diferentes, caracterizadas como opções no dinheiro, fora do dinheiro e dentro do dinheiro. 


\begin{tabular}{|c|c|c|c|}
\hline & Resumo Estatístico & $\begin{array}{l}\text { Volatilidade Implicita no } \\
\text { topo do cone de } \\
\text { volatilidade* }\end{array}$ & $\begin{array}{l}\text { Volatilidade Implicita no } \\
\text { vale do cone de } \\
\text { volatilidade } * *\end{array}$ \\
\hline \multirow[t]{4}{*}{ fora do dinheiro } & máximo & $18 \%$ & $0 \%$ \\
\hline & média & $0 \%$ & $5 \%$ \\
\hline & mínimo & $-9 \%$ & $-9 \%$ \\
\hline & \# observações & 8 & 10 \\
\hline \multirow[t]{4}{*}{ no dinheiro } & máximo & $13 \%$ & $0 \%$ \\
\hline & média & $4 \%$ & $7 \%$ \\
\hline & mínimo & $-15 \%$ & $-10 \%$ \\
\hline & \# observações & 11 & 20 \\
\hline \multirow[t]{4}{*}{ dentro do dinheiro } & máximo & $34 \%$ & $0 \%$ \\
\hline & média & $13 \%$ & $18 \%$ \\
\hline & mínimo & $-6 \%$ & $-6 \%$ \\
\hline & \# observações & 8 & 10 \\
\hline Estatística F & & 3,836 & 1,868 \\
\hline Sig. & & 0,036 & 0,169 \\
\hline
\end{tabular}

Tabela 8 - Variação entre a volatilidade implícita ao atingir níveis superiores ou inferiores ao cone de volatilidade e a volatilidade realizada até o exercício para opções da Petrobras fora do dinheiro, no dinheiro e dentro do dinheiro.

Fonte: elaboração própria

Apesar dos resultados encontrados indicarem um ganho médio maior para opções dentro do dinheiro, tanto nos casos de indicação de compra quanto indicação de venda de volatilidade, não se pode rejeitar a hipótese nula, ao nível de $1 \%$ de significância, de que os resultados médios apresentados para cada tipo de opção sejam iguais. Portanto, não existem evidências de que a utilização do cone de volatilidade apresente resultados diferentes para opções com preços de exercício diferentes.

A análise do cone de volatilidade diante do período de estresse não pôde ser realizada, porque o número de indicações de oportunidade de compra e venda de volatilidade no período de julho de 2008 até junho de 2009 foi menor que três eventos.

Portanto, a utilização do cone de volatilidade para opções da Petrobras indica que o mesmo pode contribuir com informações adicionais para a tomada de decisão nas operações de compra e venda de volatilidade, pois mostra de forma confiável se as opções estão caras ou baratas.

Mesmo que os resultados para diferentes preços de exercício não terem sido tão robustos como no caso da Vale, a existência do cone de volatilidade para as opções da Petrobras e o consequente comportamento enviesado da volatilidade das mesmas é mais uma indicação de que os preços dos ativos financeiros não se comportam da maneira eficiente como previsto pela Teoria Moderna de Finanças.

\section{Conclusões}

O presente trabalho procurou testar a utilização do cone de volatilidade no mercado de opções brasileiro com o objetivo de avaliar se o mercado de opções é inteiramente eficiente quando a volatilidade é operada em percentuais muito altos ou muito baixos, ou seja, se a posição da volatilidade implícita em relação ao cone de volatilidade pode gerar informações úteis e confiáveis para averiguar se a opção está com um preço alto ou baixo. Para isto foram utilizadas séries históricas da Vale e Petrobras no período 03/01/2005 até 30/06/2009.

Inicialmente, foram realizados testes sobre o histórico de volatilidade implícita calculada pelo método apresentado em Beckers (1981) para avaliar se a mesma segue um processo estocástico 
de reversão à média. A estacionariedade das séries de volatilidade é uma das principais premissas para testar a existência do cone de volatilidade. Através do teste da raiz unitária foi evidenciado que tanto para a Petrobras quanto para a Vale a série de volatilidade é estacionaria. Portanto, assim como em Dixit, et al. (2007), existem evidências de que a volatilidade implícita segue um processo estocástico de reversão à média.

Os resultados apresentados indicam que a utilização do cone de volatilidade apresenta retorno médio positivo tanto para operações de compra como de venda de volatilidade para opções da Vale e da Petrobras. Este resultado está em linha com as evidências apresentadas em Burghardt e Morton (1990), confirmando a hipótese de que a distribuição da volatilidade histórica pode ser usada para melhorar as previsões da volatilidade futura. Nos momentos em que a volatilidade implícita esteve negociada em níveis muito altos ou muito baixos, foi evidenciado que a volatilidade implícita é um previsor da volatilidade realizada e que nestes casos, o mercado de opções não é inteiramente eficiente, corroborando o estudo apresentado em Jorion (1995).

Isto pode ser considerado uma importante contribuição deste trabalho, pois demonstra, para o mercado de opções, que a formação de preço não se comporta de maneira racional, conforme previsto pela Hipótese dos Mercados Eficientes. Estes desvios do preço justo ocasionam não só oportunidades de arbitragem para os operadores, o que pode gerar lucros anormais, como também proporcionam uma gestão de risco mais eficiente para aqueles que conseguem prever tais anomalias. Isto confirma resultados já obtidos para diferentes ativos financeiros e amplamente estudados na emergente área de Finanças Comportamentais (Ackert e Deaves, 2010).

Os efeitos do sorriso de volatilidade sob o cone de volatilidade também foram analisados neste trabalho. Os resultados evidenciam que existem diferenças significativas na comparação da volatilidade implícita de opções fora do dinheiro, no dinheiro e dentro do dinheiro com o cone de volatilidade para as opções da Vale, sendo que os melhores resultados foram para opções dentro do dinheiro. Não houve diferença significativa no caso das opções fora do dinheiro, no dinheiro e dentro do dinheiro da Petrobras.

Trabalhos futuros poderiam aprimorar os resultados do cone de volatilidade, ao utilizar dados de transações intradiárias. Desta forma, os efeitos de ausência de sincronia entre os mercados de ações e opções para o cálculo da volatilidade implícita poderiam ser eliminados. Havendo reversão à média ao longo do dia, seria possível montar operações de day-trade de compra e venda de volatilidade. Além disto, poderia ser analisado empiricamente se as anomalias comprovadas neste estudo também persistem com dados de alta frequência.

\section{Referências Bibliográficas}

ACKERT, L. F.; DEAVES, R. Behavioral Finance - Psychology, Decision-Making, and Markets. Mason: South-Western Cengage Learning, 2010.

BECKERS, S. Standard Deviations Implied in Option Prices as Predictors of Future Stock Price Variability. Journal of Banking and Finance, v. 5, n.3, p. 363-382, 1981.

BLACK, F.; SHOLES, M. The Pricing of Options and Corporate Liabilities. Journal of Political Economy, v. 81, n. 3, p. 637-659, 1973.

BURGHARDT, G.; MORTON, L. How to Tell if Options are Cheap. The Journal of Portfolio Management, v. 16, n. 2, p. 72-78, 1990. 
DESTERRO, M. P. Modelagem do smile de volatilidade implícita de opções de compra de dólar no mercado brasileiro - aproximação sticky-delta. Dissertação (Mestrado em Administração) COPPEAD. Rio de Janeiro: UFRJ, 2003.

DIXIT, A.; YADAV, S.S.; JAIN, P.K. Testing the expectations hypothesis on the term structure of volatilities implied by index options: study of the Indian securities market. Journal of Financial Management and Analysis, v. 20, n. 2, p. 38-55, 2007.

FIGLEWSKI, S. Forecasting Volatility. Financial Markets, Institutions \& Instruments, v. 6, n. 1, p. 1-88, 1997.

HODGES, S.; TOMPKINS, R. Volatility cones and their sampling properties. Journal of Derivatives, v. 10, n. 1, p. 27-42, 2002.

JORION, P. Predicting Volatility in the Foreign Exchange Market. Journal of Finance, v. 50, n. 2, p. 507-528, 1995.

JUNIOR, G.G. Sorriso da volatilidade nas opções de compra de Telemar PN usando dados intradiários. Dissertação (Mestrado em Administração) - COPPEAD. Rio de Janeiro: UFRJ, 2007.

RODRIGUES, A, F. A utilização de dados intradiários para a construção de modelos de previsão de volatilidade: uma comparação entre a variância realizada e a amplitude realizada. Dissertação (Mestrado em Administração) - COPPEAD. Rio de Janeiro: UFRJ, 2006.

STEIN, J. Overreaction in the Options Market. Journal of Finance, v. 44, n. 4, p. 1011-1023, 1989.

XU, X.; TAYLOR, S. J. The term structure of volatilities implied by foreign exchange options. Journal of Financial and Quantitative Analysis, v. 29, n. 1, p. 57-74, 1994. 